\title{
The BABAR Event Building and Level-3 Trigger Farm Upgrade
}

\author{
R. Jacobsen \\ Lawrence Berkeley National Laboratory and University of California, Berkeley, California 94720 \\ G. Dubois-Felsmann, I. Narsky \\ California Institute of Technology, Pasadena, California 91125 \\ E. Rosenberg \\ lowa State University, Ames, lowa 50011-3160 \\ B. Franek \\ Rutherford Appleton Laboratory, Chilton, Didcot, Oxon, OX11 OQX United Kingdom \\ R. Bartoldus, J. Hamilton, D. Kotturi, S. Luitz, C. O'Grady, A. Perazzo, R. Rodriguez, A. Salnikov, M. \\ Weaver, M. Wittgen \\ SLAC, Stanford, CA 94025, USA \\ S. Dasu \\ University of Wisconsin, Madison, Wisconsin 53706
}

for the BABAR Computing Group

\begin{abstract}
The BABAR experiment is the particle detector at the PEP-II B-factory facility at the Stanford Linear Accelerator Center [1. During the summer shutdown 2002 the BABAR Event Building and Level-3 trigger farm were upgraded from 60 Sun Ultra-5 machines and 100MBit/s Ethernet to 50 Dual-CPU 1.4GHz Pentium-III systems with Gigabit Ethernet. Combined with an upgrade to Gigabit Ethernet on the source side and a major feature extraction software speedup, this pushes the performance of the BABAR event builder and L3 filter to $5.5 \mathrm{kHz}$ at current background levels, almost three times the original design rate of $2 \mathrm{kHz}$. For our specific application the new farm provides 8.5 times the CPU power of the old system.
\end{abstract}

\section{THE BABAR DATA ACQUISITION SYSTEM}

The primary data handling components of the BABAR data acquisition system are the Online Data Flow (ODF) 2] and the Online Event Processing (OEP) [3]. ODF is the real-time system for trigger distribution, readout of the front end electronics, feature extraction and multi-stage event building. OEP provides near-real-time processing of fully built events: Level-3 software trigger, fast data quality monitoring, calibration processing and dispatch to data logging.

Figure 1 gives an overview of the BABAR data acquisition system.

Sampling and analog-to-digital conversion takes place in the front end electronics, mounted directly on or close to the subdetectors. From there, data is sent to "Read-Out-Modules" (ROMs) which are 9u VME modules that combine a commercial PowerPC single board computer running the VxWorks operating system with custom-built components. The number of VME crates and ROMs in the crate depends on design and data load of the subdetector. The ROMs apply detector-specific feature extraction algorithms and then transfer their event contributions via the VME bus to the ROM in slot 0 of their crate. This is the first stage of event building. The next stage is to combine all slot- 0 ROM contributions of an event by sending them to the same Level-3 trigger farm node via Ethernet. The network event builder uses a UDPbased protocol, does not attempt to retransmit lost packets and employs a simple window-based flow control mechanism. Events are sent to a quasi-random sequence of farm nodes by using a hash value based on the event time stamp.

In the farm node, the Level-3 filter algorithms are applied on the complete event, events that pass the selection are sent to a central logging server where they are written to disk files. These files are subsequently transferred to the SLAC computer center for permanent tape storage and near-real-time full reconstruction.

The initial design goal was $2 \mathrm{kHz}$ of $30 \mathrm{kByte}$ events to be read out with negligible dead time from the front end electronics, feature extracted, built and shipped to the Level-3 trigger that would select $100 \mathrm{~Hz}$ of events for logging and permanent storage. With the original farm of $32330 \mathrm{MHz}$ Sun Ultra-5 machines the system did indeed reach $2 \mathrm{kHz}$, limited by the amount of CPU time available to the Level-3 software trigger.

\section{PLANNING THE UPGRADE}

\subsection{Reasons for Upgrading}

A farm upgrade became necessary for the following reasons:

- The projected PEP-II luminosities and trigger rate extrapolations require the data acquisition system to handle higher rates and larger event sizes, in addition, operational experience showed 


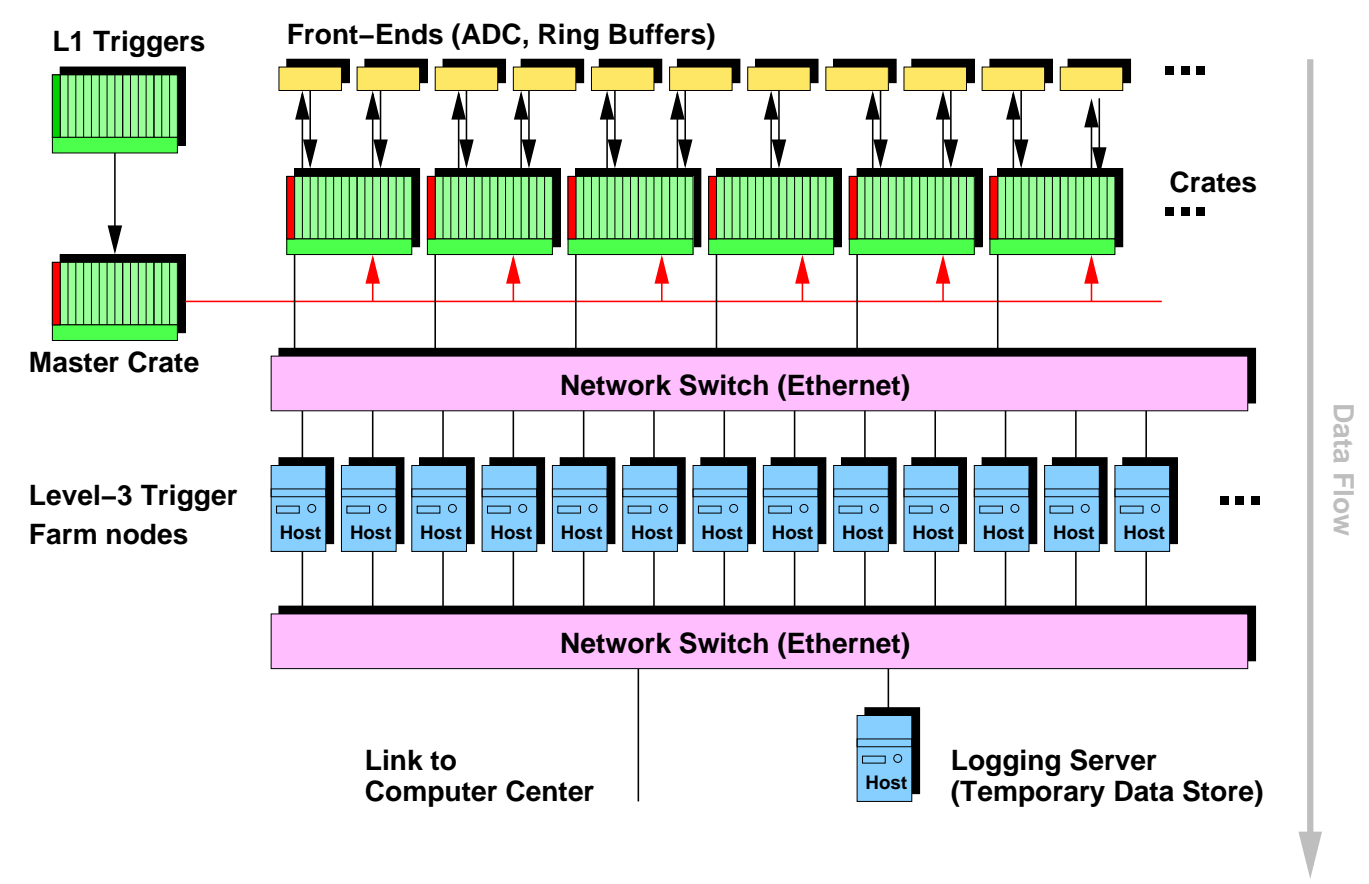

Figure 1: Overview of the BABAR Data Acquisition System

that headroom of a factor of two is desirable to handle temporary high background conditions.

- Having more CPU for more advanced Level-3 trigger algorithms will facilitate to keep the logging rate low.

- The Sun Ultra-5 machines were reaching their end of life, showing an increasing number of hardware failures.

To be able to safely handle the projected trigger rates until at least 2005, it was decided that an upgrade should increase the farm CPU capacity by a factor of about 10 and that the 100MBit/s Ethernet connections in the network event builder should be upgraded to Gigabit Ethernet.

\subsection{Requirements and Upgrade Options}

Any upgrade option had to meet the following basic requirements:

- Run one of the two operating systems supported as BABAR software platforms: RedHat Linux on i386 or Solaris/SPARC

- Hardware must be available during the planning phase (fall 2001) so that performance measurements could be obtained and to prevent product availability from impacting the rigid deployment schedule during the Summer 2002 shutdown.
Some options had been ruled out a priori, because they were expected to be too different from the existing system and would have required extensive $R \& D$ and testing efforts, for example multiprocessor systems with more than 2 CPUs where the concern were symmetric multiprocessing scaling and interrupt distribution issues.

In the end, the following major options were considered:

1. Rack-mountable Sun $750 \mathrm{MHz}$ UltraSPARC-III dual-CPU systems (4 rack units high)

2. Replace Sun $330 \mathrm{MHz}$ Ultra-5 machines with rack-mountable 1-CPU $440 \mathrm{MHz}$ Sun machines (1 rack unit high), add systems as necessary and replace the farm later when faster and cheaper SPARC single or dual CPU systems became available

3. Intel 1.2Ghz Pentium-III Dual-CPU systems with Linux (1 rack unit high)

Both Sun options (1] and 2) had the advantage that no software modifications to ODF were required and that they could just be used as a drop-in replacement. However, compared to the Intel option (3), they were significantly more expensive and had much larger rack space requirements: For comparable CPU power, option 1 needed more rack space because of slower CPUs and a larger form factor, option 2 just because of the much slower CPUs. 
The Intel option had the major disadvantage to require modifications to ODF and OEP software in order to accommodate the different byte ordering. Option 2 had the additional disadvantage to be a twostep upgrade where the second step relied on not yet existing hardware.

After extensive consideration of these options, the Intel Linux option was chosen because it was expected to be the most cost-effective and long-term maintainable solution.

\section{ADAPTING THE SOFTWARE}

\subsection{Data Flow Software}

ODF hardware and software is responsible for realtime readout, data transport, feature extraction and multi-stage event building. The last stage is a network event builder implemented on top of UDP, using no retransmit and a simple end-to-end flow control protocol. The underlying link-level protocol is Ethernet. To maximize system performance, the original design required the machines on the receiving end of the network event builder to have the same byte ordering as the PowerPC embedded systems on the source side. For the network event builder, the event data payload is transparent binary data.

In order to accommodate Intel/Linux farm machines, ODF software had to be modified. To avoid wasting precious CPU time on the embedded systems, all byte swapping occurs on the farm machines. The ODF network protocol was changed so that all datagram headers are 32-bit aligned and swappable and whole datagrams (ODF headers as well as event data payload) are 32-bit byte swapped after they have been received by the farm machines. ODF software then builds the event and delivers the complete preswapped event data to OEP.

Since the ODF network protocol always uses the source byte order, it is independent of the byte order of the farm machines and allows the use of Sun or Linux machines, in principle even in a mixed farm. During testing we took advantage of this very important feature.

In parallel to upgrading the farm to Gigabit Ethernet, the embedded systems were upgraded to Gigabit Ethernet. To minimize the overhead in CPU and wall clock time for sending a packet, a custom minimal UDP protocol stack and network device driver was developed. This driver sends a 1500 byte packet in $17.3 \mu \mathrm{s}$, compared to the $66 \mu \mathrm{s}$ of the VxWorks driver that came with the Gigabit Ethernet interface card and the $100 \mu$ s needed by the $100 \mathrm{MBit}$ ethernet driver used before the upgrade.

\subsection{Online Event Processing and Level-3 Filter Software}

OEP and Level-3 filter obviously need to look at the event data. While all navigational information is 32-bit wide and aligned on 32-bit boundaries, individual containers can hold 8-bit, 16-bit and 32-bit quantities. As mentioned above, events are delivered to OEP pre-swapped, that means that the navigational information is already intact. The classes that describe the data format then know how to fix up 8-bit and 16-bit quantities, this process only happens on demand. The on-disk representation of data is always big-endian, so on little endian machines the reverse process is applied before data is written out to disk or sent over the network.

\subsection{System Software and System Management}

System management for Linux machines in the $B A B A R$ online computing infrastructure was achieved by modifying the BABAR-online-specific version of Taylor to support RedHat Linux [5] and to set up a Kickstart configuration for network installation of Linux machines. Taylor is a system post-install and configuration language developed at SLAC [4].

\section{TESTING AND HARDWARE ACQUISITION}

\subsection{Choosing Hardware}

Since time and personnel resources for testing were limited, the initial choice of hardware was made, considering experience from the SLAC windows group who was already operating a number of Dell PowerEdge 15501 rack-unit, $1.2 \mathrm{GHz}$ dual-CPU servers with Intel E1000 Gigabit Ethernet cards. We decided to use fiber-optic Gigabit Ethernet because at decision time there was no significant experience at SLAC with the cheaper copper cable Gigabit Ethernet.

A small number of machines was acquired and used in different testing modes:

- Teststand testing during software development was used to develop the software, set up the system software infrastructure and verify the basic suitability of the machines for the task.

- "Parasitic" testing in the real system was used to find problems with system stability or other operational issues. The connection-less and byte order independent event builder protocol allowed to use the port monitoring feature of the event building switch to send real-time copies of 
datagrams to a small number of machines being tested. In this mode, the switch was configured to send copies of all packets addressed to a Sun farm node to the interface of a Linux node that had been set up with the same MAC and IP addresses as the original node but had been blocked from sending any replies back.

To ensure proper operation, two machines were run in this mode for several months, no problems were found.

- Small-scale testing of up to six new machines in the real system.

By the time of the purchase decision, the tested hardware was no longer available and we had to go through another testing cycle with the successor model (Dell PowerEdge 1650). At that time it was decided to use $1.4 \mathrm{GHz}$ systems instead of $1.2 \mathrm{GHz}$.

Compared to the $330 \mathrm{MHz}$ UltraSPARC-II baseline, our application runs 2.75 times as fast on a $1.4 \mathrm{GHz}$ Pentium-III CPU. Because of limitations in the number of Gigabit Ethernet switch ports and cost issues, we decided to purchase 50 new machines in the final configuration:

- Dual-Pentium-III, $1.4 \mathrm{GHz}$

- 1GByte RAM

- optical Intel E1000 Gigabit Ethernet card

- single power supply

- no remote lights-out management capabilities (during data taking there are always operators in the control room who can manually power cycle a machine if necessary)

Compared to the old Sun farm the new farm provides 8.5 times the CPU power.

\section{DEPLOYMENT AND RESULTS}

During a 4-month shutdown in summer 2002, the 50 new machines were installed in 3 water-cooled racks in the $B A B A R$ electronics hut. Since the Dell rack rails could not easily be made fit into the existing racks, pairs of machines were stacked on regular rack shelves. The electronics hut is accessible during data taking, so it was decided to save the cost of a remote power control or reset system and simply install serial lines to the console ports. New modules with a total of $80 \mathrm{Gi}-$ gabit Ethernet ports were installed in the Cisco 6509 event building switch. To satisfy subdetector data acquisition needs during the shutdown, a part of the old farm was kept in place and operational. Installation and startup of the new farm was very smooth, one machine was broken on arrival and another machine failed after one week of operation.

We did not encounter other major problems and since the start of BABAR Run 3 in November 2002 the new system is used in production. After additional improvements to the feature extraction code the system is now capable of handling $5.5 \mathrm{kHz}$ Level-1 trigger rate at $30 \mathrm{kByte}$ event size and nominal background rates, limited by the feature extraction code in the ROMs.

We did not observe any stability problems with the Linux farm machines.

\section{CONCLUSIONS}

The upgrade of the BABAR data acquisition system to Linux and Gigabit Ethernet has been successful and improved the maximum rate capability at nominal backgrounds to $5.5 \mathrm{kHz}$.

\section{ACKNOWLEDGEMENTS}

We would like to thank everyone who contributed to the upgrade project and helped to actually make it happen on time.

\section{References}

[1] By BABAR Collaboration (B. Aubert et al.), "The BaBar Detector", Nucl.Instrum.Meth. A479:1116,2002

[2] R. Claus et al., "Development of a Data Acquisition System for the BABAR CP Violation Experiment",Presented at 11th IEEE NPSS Real Time Conference (Santa Fe 99), Santa Fe, NM, 14-18 Jun 1999.

[3] G.P Dubois-Felsmann et al, "Flexible Processing Framework for Online Event Data and Software Triggering", IEEE Trans.Nucl.Sci.47:353-357,2000

[4] www.slac.stanford.edu/comp/unix/taylor.html

[5] www.redhat.com 